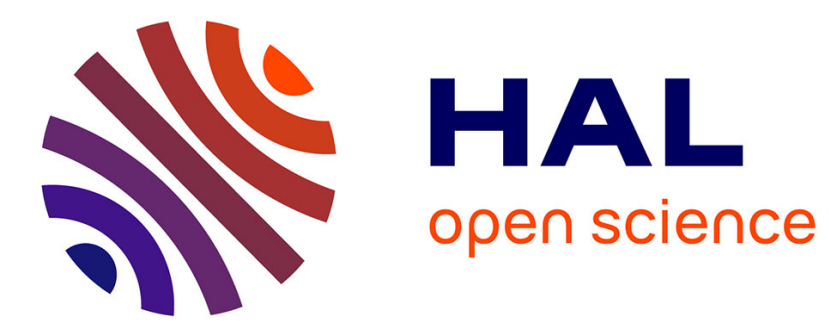

\title{
Self-Assembly of CdSe Nanoplatelets into Giant Micrometer-Scale Needles Emitting Polarized Light
}

Benjamin Abécassis, Mickael D. Tessier, Patrick Davidson, Benoit Dubertret

\section{To cite this version:}

Benjamin Abécassis, Mickael D. Tessier, Patrick Davidson, Benoit Dubertret. Self-Assembly of CdSe Nanoplatelets into Giant Micrometer-Scale Needles Emitting Polarized Light. Nano Letters, 2014, 14 (2), pp.710 - 715. 10.1021/nl4039746 . hal-01596125

\section{HAL Id: hal-01596125 \\ https://hal.science/hal-01596125}

Submitted on 9 Nov 2020

HAL is a multi-disciplinary open access archive for the deposit and dissemination of scientific research documents, whether they are published or not. The documents may come from teaching and research institutions in France or abroad, or from public or private research centers.
L'archive ouverte pluridisciplinaire HAL, est destinée au dépôt et à la diffusion de documents scientifiques de niveau recherche, publiés ou non, émanant des établissements d'enseignement et de recherche français ou étrangers, des laboratoires publics ou privés. 


\title{
Self-assembly of CdSe nanoplatelets into giant
}

\section{micron-scale needles emitting polarized light}

\author{
Benjamin Abécassis, ${ }^{*} \dagger$ Mickael D. Tessier, ${ }^{\dagger}$ Patrick Davidson, ${ }^{\dagger}$ and Benoit \\ Dubertret $^{*,+}$
}

Laboratoire de Physique des Solides, Univ. Paris-Sud, CNRS, UMR 8502, F-91405 Orsay Cedex, France, and Laboratoire de Physique et d'Etude des Matériaux, CNRS, Université Pierre et Marie Curie, ESPCI, 10 rue Vauquelin, 75005, Paris, France.

E-mail: benjamin.abecassis@u-psud.fr; benoit.dubertret@espci.fr

${ }^{*}$ To whom correspondence should be addressed

${ }^{\dagger}$ Laboratoire de Physique des Solides, Univ. Paris-Sud, CNRS, UMR 8502, F-91405 Orsay Cedex, France

${ }^{\ddagger}$ Laboratoire de Physique et d'Etude des Matériaux, CNRS, Université Pierre et Marie Curie, ESPCI, 10 rue Vauquelin, 75005, Paris, France. 


\section{Abstract}

We report on the self-assembly of colloidal CdSe nanoplatelets into micrometers long anisotropic needle-like superparticles (SPs) which are formed in solution upon addition of an antisolvant to a stable colloidal dispersion. Optical fluorescence microscopy, transmission electron microscopy and small angle $\mathrm{X}$-ray scattering provide detailed structural characterization and show that each particle is composed of $10^{6}$ nanoplatelets organized in highly aligned columns. Within the SPs, the nanoplatelets are stacked on each other to maximize the contact surface between the ligands. When deposited on a substrate, the planes of the platelets are oriented perpendicularly to its surface and the SPs exhibit polarized emission properties.

Keywords : CdSe; Nanoplatelets; nanosheets; self-assembly; SAXS; TEM 
The self-assembly of nanocrystals into larger, ordered microstructures is a current matter of interest in the field of nanotechnology. ${ }^{1,2}$ Since new collective physical properties can eventually emerge from these supra-crystals, resulting from the coupling between the building blocks, important efforts are deployed in order to set-up efficient procedures for the synthesis of such structures. This quest is also motivated by the important applicative promises of nanoparticles which will only come true if a fine and tunable control over their three-dimensional long-range organization is achieved.

Among anisotropic semi-conducting nanoparticles, the self-assembly of CdSe quantum rods in solution or on a substrate has already been achieved using a variety of techniques such as application of electric fields, ${ }^{3}$ depletion interaction, ${ }^{4}$ slow diffusion of an antisolvant ${ }^{5}$ or evaporation on a solid substrate. ${ }^{6}$ Branched CdSe nanocrystals have also been assembled into hierarchical superlattices in solution. ${ }^{7}$ Recently, CdSe-CdS nanorods were self-assembled into large crystalline superparticles which exhibit anisotropic photoluminescence. ${ }^{8,9}$ As it is much easier to manipulate microparticles than nanoparticles, this method is an elegant way to orient the nanorods on a surface and to exploit their orientation-dependent physical properties in devices.

The 2D counterpart of colloidal nanorods, i.e., colloidal semi-conducting nanoplatelets (NPs) have not received much attention yet. These nanoparticles have recently been discovered ${ }^{10,11}$ and show outstanding optical properties such as tunable emission wavelength, fast carrier recombination and high quantum yield. ${ }^{12}$ They are synthesized in solution, through the reaction of cadmium and selenium precursors in the presence of ligands (long chain carboxylic acids) which adsorb on the cadmium rich (001) surface of the nanoparticle and make them dispersible in apolar solvants such as hexane or toluene. While it has been observed that cadmium acetate is a necessary component for the two-dimensional growth to occur, the relative concentrations in platelets of different thicknesses and their lateral extension depend on other parameters of the synthesis such as the reaction temperature, the concentration in precursors and ligands or the injection speed of the metal precursors. Three populations of nanoplatelets have been synthesized to date which emit at 463, 513 and $550 \mathrm{~nm}$ and differ in thickness by a CdSe monolayer (ML), i.e., half a lattice unit. This 
corresponds to platelet thicknesses of $3 \mathrm{CdSe} \operatorname{MLs}(\simeq 0.9 \mathrm{~nm}), 4 \mathrm{MLs}(\simeq 1.2 \mathrm{~nm})$ and $5 \mathrm{MLs}(\simeq$ $1.5 \mathrm{~nm}) .{ }^{13}$ More importantly, colloidal solutions containing only one population of platelets can be prepared.

From the self-assembly point of view, it has recently been pointed out that $2 \mathrm{D}$ systems could be of interest since the interaction between the ligands covering the particle is far more effective when the curvature of the nanoparticle is as low as possible. ${ }^{14,15}$ This can easily be understood by considering the interaction between two particles with ligands bound on their surface. When spherical or rodlike particles approach, only a small fraction of the bound ligands interact as their orientation follows the curved geometry of the nanoparticle. On the contrary, when two plate-like particles are brought together, all the ligands on one face of one particle come in close contact with all the ligands on the opposing face of the other. Hence, in this geometry, the ligand-ligand interaction is of particular importance and since it is easily tunable through physico-chemical parameters, we expect that the assembly of platelets will show a rich behavior.

Several examples of colloidal nanoplatelets assembly have appeared in the literature. For example, Murray et al. proved the potential of the liquid interface self-assembly to direct the formation of 2D long-ranged nanoplatelets superlattices. ${ }^{16,17}$ Korgel et al. have shown that $\mathrm{Cu}_{2} \mathrm{~S}$ nanoplatelets self-assemble in columnar assemblies on substrates. ${ }^{18}$ Long anisotropic superstructures have been observed in solutions of the same nanoparticles by a different group. ${ }^{19}$

In a previous study, we have shown that the stacking of CdSe nanoplatelets in solution can have important consequences on their emission properties. Indeed, the interaction between the nanoplatelets within a stack induces the appearance of a phonon-replica line in their emission spectrum at low temperature. ${ }^{20}$ Here, we show that the addition of an anti-solvant to a stable dispersion of CdSe nanoplatelets in hexane triggers the formation of anisotropic supra-crystals whose length can reach several micrometers. The platelets stack in a 1D-fashion one on top of each other and exhibit a columnar order within the superparticle floating freely in solution. Furthermore, the platelets are all oriented perpendicular to the long axis of the needle and hence, they display polar- 
ized light emission.
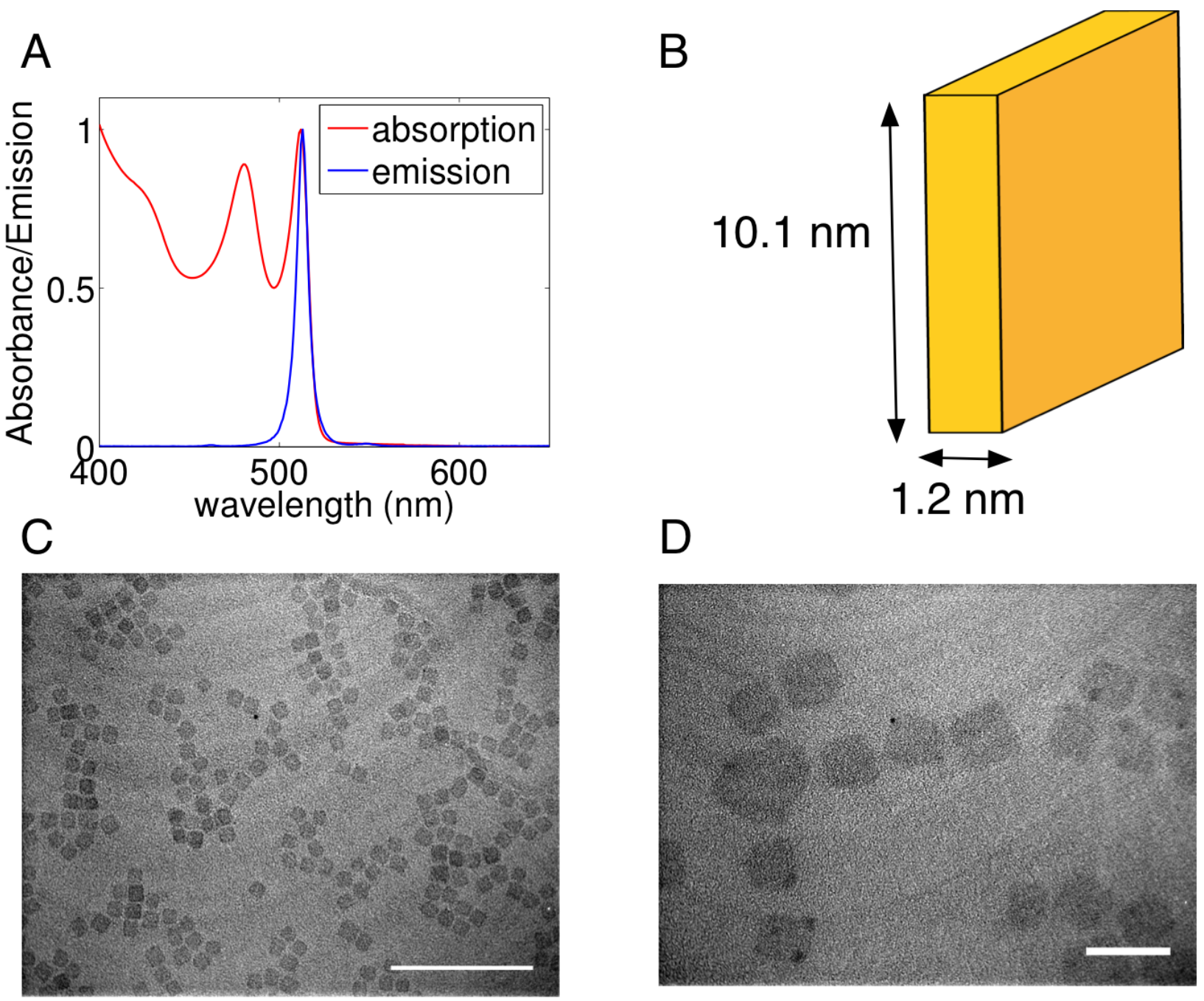

Figure 1: A) Absorption and emission spectra of the CdSe nanoplatelets. B) Schematic representation of the nano-platelets. C) and D) TEM images. Scale bars measure $100 \mathrm{~nm}$ and $20 \mathrm{~nm}$ respectively.

We synthesized CdSe nanoplatelets as described in the methods section following a modification of a previously reported synthesis. ${ }^{10,12}$ After purification, we obtain a dispersion of NPs the absorption and emission spectra of which are shown on figure 1.a. The two sharp maxima at $513 \mathrm{~nm}$ and $480 \mathrm{~nm}$ in the absorption spectrum correspond respectively to the electron-heavy hole and electron-light hole transitions due to the quantum confinement of the electron/hole pair in the potential well created by the limited thickness of the platelets. As no other peak is visible, 
the dispersion only contained one population of 4 MLs CdSe NPs. The large excess of oleic acid added at the end of the synthesis ensures that these ligands coat the faces of the NPs. Electron microscopy investigation of the NPs (figure 1) shows that they have a roughly square shape with a monodisperse size distribution. The edges measure on average $10.1 \mathrm{~nm}$ with a standard deviation of $1.15 \mathrm{~nm}$ (based on measurement of 300 nanoparticles).
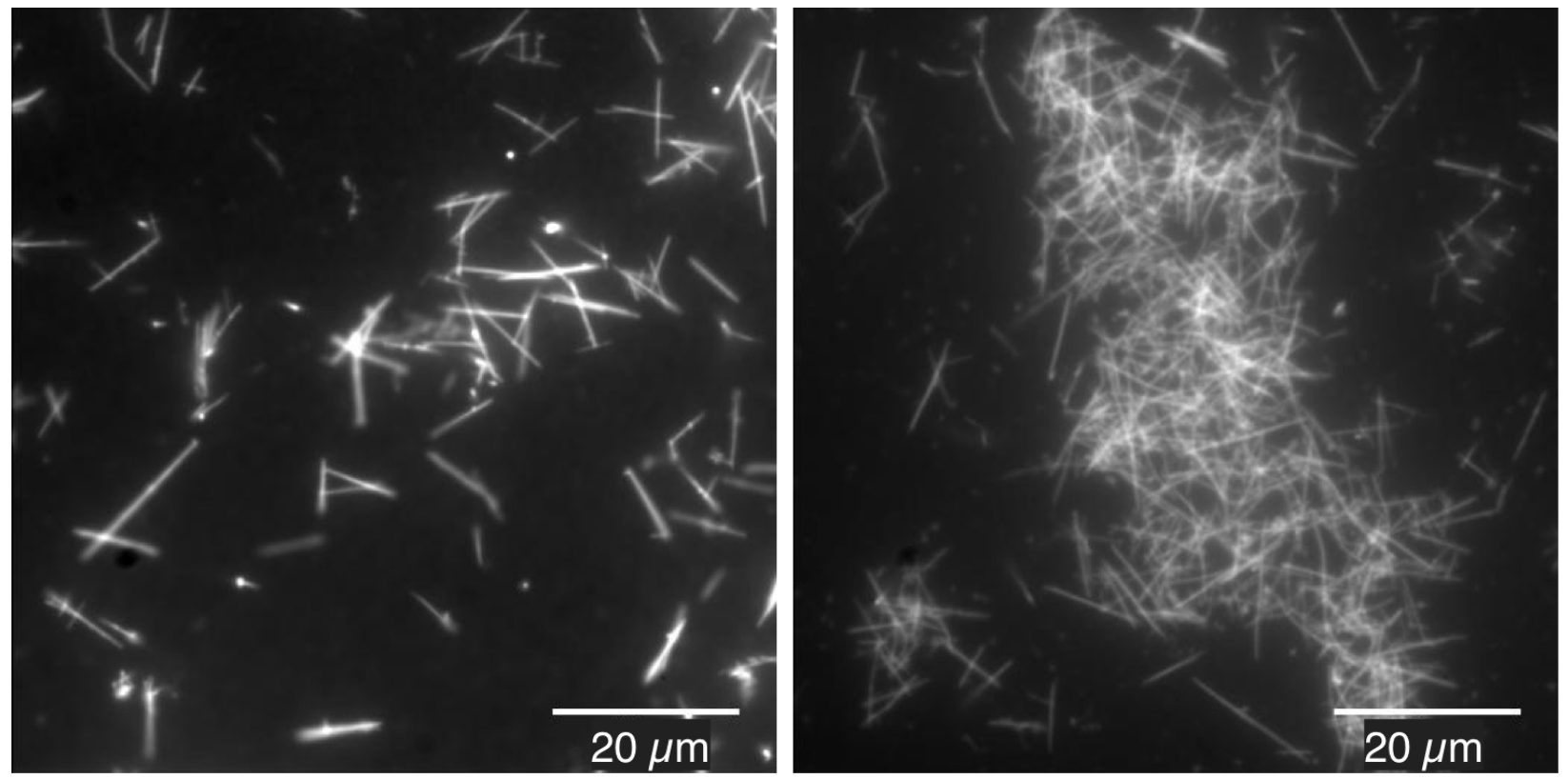

Figure 2: Fluorescence microscopy of the super-particles deposited on a glass slide.

When a small amount of ethanol is added to the dispersion of NPs in hexane, the solution becomes slightly turbid after a few seconds, indicating the presence of large particles which scatter light. Fluorescence optical microscopy observation of the dispersion indicates the presence of long anisotropic particles free-floating in solution (figure 2).

These highly fluorescent superparticles can reach $20 \mu \mathrm{m}$ in length while their width is beyond the resolution of the optical set-up. Transmission Electron Microscopy confirms the formation of anisotropic SPs composed of individual nanoplatelets. TEM images of fresh (a few hours) samples display fiber-like superparticles composed of a few subunits separated by regions where the density of nanoparticles is smaller (figure 3). In some cases, kinks are visible at these places (see supplementary materials). However, in TEM images of aged (a few months) samples (supplementary 


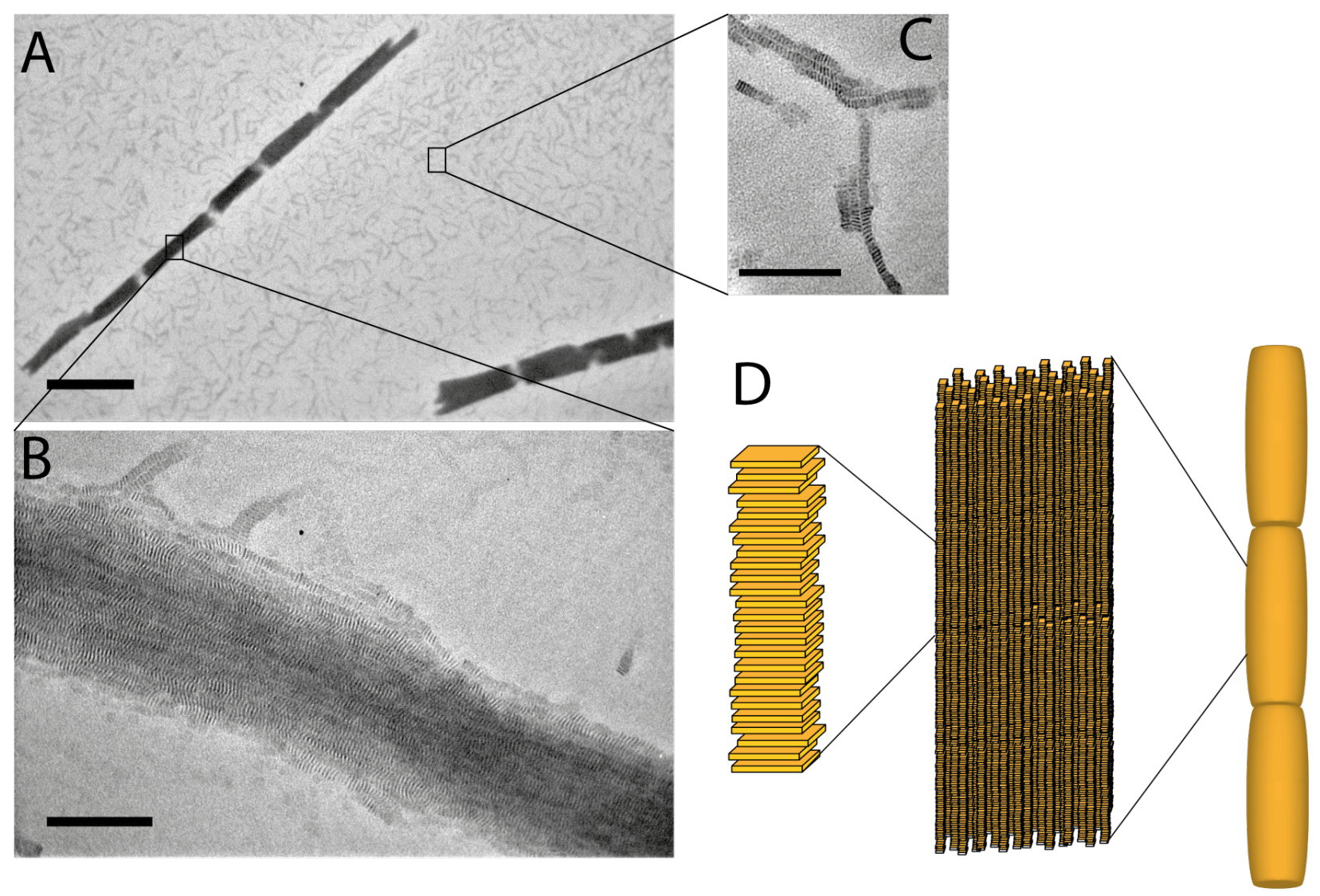

Figure 3: TEM images of the superparticles A) Low magnification image showing an entire superparticle. Scale bar is $1 \mu \mathrm{m}$. B) Higher magnification image showing the stacking of the nanoplatelets within a superparticle. Scale bar is $100 \mathrm{~nm}$. C) Subunits which can seen besides the superparticles. Scale bar is $100 \mathrm{~nm} \mathrm{D)} \mathrm{Scheme} \mathrm{representing} \mathrm{the} \mathrm{hierarchical} \mathrm{structure} \mathrm{of} \mathrm{the}$ superparticles. 
materials), long defect-less needles are observed. We suspect the defects to arise from a drying instability occurring during the preparation of the TEM grid. This difference might be due to the fact that long-term rearrangements of platelets within the aged SPs provide them with a higher density and rigidity, which prevents them from breaking upon drying constraints.

Within the SPs, the nanoplatelets are organized in a highly aligned columnar structure where the principal axis is perpendicular to the plane of the nanoplatelets. In the two lateral directions, between 10 and 20 columns of stacked NPs stand side by side within the superparticles whose widths are comprised between 150 and $300 \mathrm{~nm}$. Based upon these dimensions, we can infer that each SP is made of $\simeq 10^{6}$ nanoplatelets. We note that, besides the superparticles, smaller ensembles consisting of a more limited number of stacked nanoplatelets (from 5 to 100) are also visible on the TEM grid (figure 3.c and supplementary materials).

To further characterize the order within the superparticles, synchrotron Small Angle X-ray Scattering (SAXS) experiments were performed. Figure 4 shows a SAXS pattern of the dispersion of SPs. The pattern being isotropic, there is no preferential orientation of the micro-needles in solution.

A series of three Bragg peaks is visible at wave vectors $\mathrm{q}=1.228,2.445$ and $3.651 \mathrm{~nm}^{-1}$ corresponding to the 001,002 and 003 stacking reflections of a columnar structure with period $5.11 \mathrm{~nm}$. At smaller wave-vectors, two less intense peaks are observed at $\mathrm{q}=0.390$ and 0.733 $\mathrm{nm}^{-1}$. These peaks correspond to a period of $16.1 \mathrm{~nm}$ and can therefore be attributed to lateral ordering of the nanoplatelet columns within the superparticles. Since the order is of much longer range in the 001 direction than in the perpendicular plane, the corresponding Bragg peaks are much more intense. The SAXS pattern thus confirms the TEM examination of the sample and is consistent with a columnar structure where positional order is much more long-ranged in the principal direction of the superparticle.

Finally, we studied the polarization of the emission of the superparticles. Since the nanoplatelets are anisotropic, we expect that they emit light polarized in their plane. To test this prediction, the platelets need to be oriented perpendicular to a substrate. Because all the nanoplatelets, in a given 
superparticle, stand oriented perpendicular to the principal direction of the microneedle, the light emitted by the superparticles should be linearly polarized.

We used an epifluorescence set-up comprising a polarizing beam splitter which allowed us to visualize on the same image both horizontal and vertical components of the light emitted by the superparticles. As seen on figure 5, the needles oriented perpendicular to the polarizer appear brighter than those oriented parallel to it. The light emitted by the superparticles is strongly polarized in the direction perpendicular to their long axis. This also shows that an individual nanoplatelet emits light polarized in its plane. Since we collect half of the light intensity polarized in the plane of the platelets, the degree of polarization can be quantified by a polarization factor $r=\left(2 I_{\perp}-I_{\|}\right) /\left(2 I_{\perp}+I_{\|}\right)$where $I_{\|}$is the intensity polarized parallel to the needles. This factor reaches $71 \%$ for the superparticle on figure 5.b. This value is of the same order of magnitude as the polarization factor measured on individual quantum $\operatorname{dots}^{21}$ and a certain degree of depolarization is probably due to some orientation disorder of the nanoplatelets within the superparticles. Recently, a polarization factor of $86 \%$ has been measured on superparticles of CdSe quantum rods ${ }^{9}$ but, in this case, the measurement was made at a fixed wavelength corresponding to the maximum photoluminescence of the rods whereas we measured an intensity averaged on all the wavelengths passing through the dichroic mirror.

We now turn to the discussion of the formation of the superparticles. The precise mechanism by which the superparticles form is still under systematic investigation and will be the topic of a future publication. Nevertheless, we already report here that several key factors influence the quality of the superparticles, in terms of aspect ratio and degree of orientational order. First, the shape of the nanoplatelets is a crucial parameter. Only nanoplatelets with well defined edges selfassemble into superparticles. Also, the cross-section of the nanoplatelets has to be square and not rectangular to yield long superparticles. This is likely a simple geometrical effect; perfectly square nanoplatelets pack more efficiently into dense superparticles than particles of more complicated shapes. Second, the assembly pathway is also of importance. As mentioned above, the formation of these superparticles is triggered by the addition of ethanol to a hexane solution of nanoplatelets. 


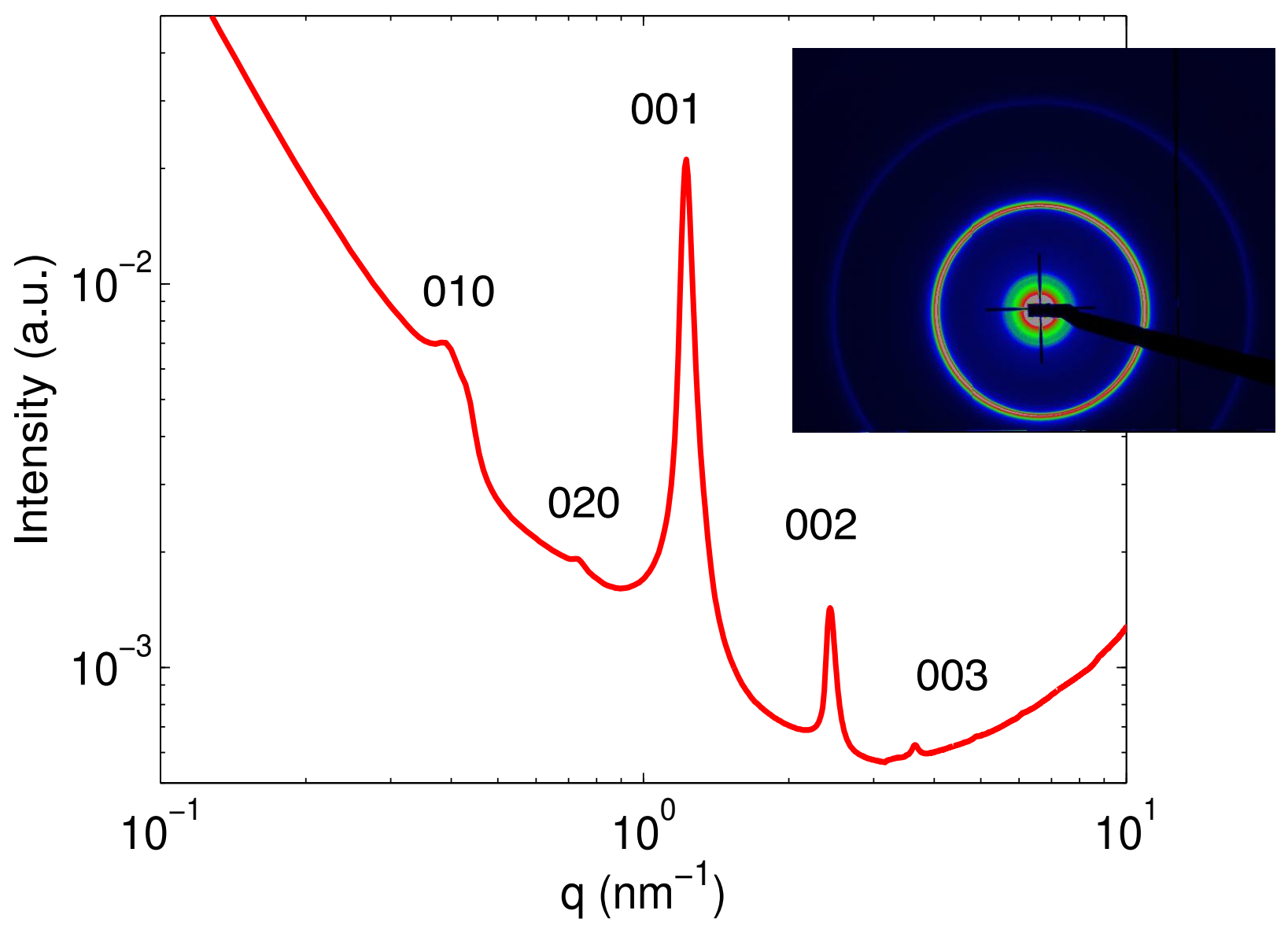

Figure 4: Small Angle X-ray Scattering pattern of the superparticles in a glass capillary. Inset: image of the scattered intensity in false colors. 
Ethanol is indeed known to induce aggregation of colloidal nanocrystals in apolar solvent. It has been shown by NMR that ethanol molecules replace oleic acid at the surface of quantum dots, ${ }^{22}$ which affects the interaction potential between the surfaces of the platelets. The steric repulsion imposed by the oleic acid brush is then suppressed and the nanoplatelets can approach each other more closely. Another effect could also explain why the addition of ethanol leads to the formation of the superparticles. Ethanol is a bad solvent for the aliphatic chain of oleic acid. Hence, when the proportion of ethanol is increased, contact between the brushes of two different platelets will be energetically favored. In other terms, particle/particle contacts (via the bound ligands) are more and more favored as the proportion of bad solvent is increased. The SAXS measurements yield a stacking period of $5.1 \mathrm{~nm}$ within the superparticles. With a thickness of $1.2 \mathrm{~nm}$ for the platelet, we can deduce that the surfaces of the platelets are $3.9 \mathrm{~nm}$ apart. This distance is consistent with the presence of oleic acid molecules at the surface of the nanoplatelets since their complete replacement by ethanol would yield a smaller stacking period. On the other hand, a fully stretched oleic acid molecule is $2.5 \mathrm{~nm}$ long, which shows that the aliphatic chains are either interdigitated or collapsed. We also noted that the amount of ethanol needed to obtain the superparticles varies from batch to batch and depends on the purification protocol of the nanoplatelet dispersion. This is likely due to the variation of the quantity of free oleic acid present in the dispersion at the end of the purification process. Adding oleic acid tends to stabilize the dispersion and delays the onset of NP aggregation. Repeated exposure of CdSe NPs to air leads to the destabilization of the dispersion and the appearance of a precipitate. The colloidal stability of the NPs and hence, their selfassembly, is very sensitive both to the concentration of oleic acid in solution and to trace amounts of water that is likely to adsorb at the particle surface and modify the interaction potential between the NPs. The kinetics of self-assembly may also be altered by the presence of water through the friction properties of the ligand monolayer. This effect has indeed been evidenced using a Surface Force Apparatus in dodecane dispersions of surfactant-coated $\mathrm{ZnS}$ nanocrystals. ${ }^{23,24}$ Fine-tuning of the size, structure and processability of the SPs will only be achieved through both the continuous refinement of the synthesis and purification protocol and the control of post-synthesis history 


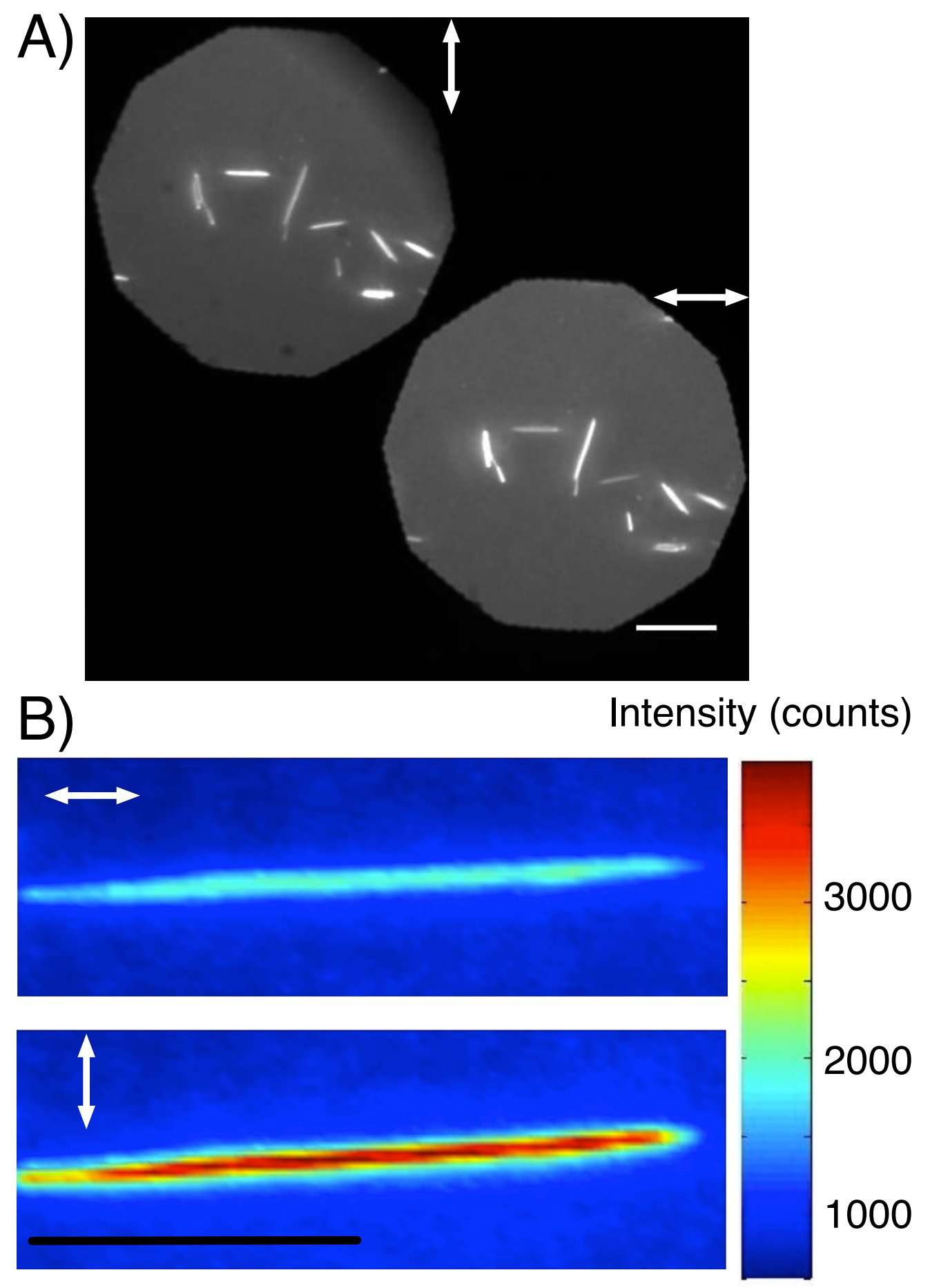

Figure 5: A) Epifluorescence measurement of microneedles as a function of the polarization direction. Scale bar is $15 \mu \mathrm{m}$. B) Fluorescence intensity in false color for a single microneedle depending on the polarization direction. Scale bar is $10 \mu \mathrm{m}$. 
of the colloidal dispersion.

Finally, we can draw an analogy between these architectures and supra-molecular assemblies of planar $\pi$-conjugated molecules which also self-assemble into long anisotropic belts. ${ }^{25-27}$ More than their similar structures, these assemblies share also a common synthetic pathway since they are both obtained through addition of an anti-solvant to a solution of dispersed building blocks. A major difference lies in the fact that the nanoplatelets used here are one order of magnitude larger in size. Molecules being small (as compared to nanoparticles), their diffusion is fast and the system can explore numerous configurations until it finds the minimum of free energy. In other terms, these systems are, most of the time, at thermodynamic equilibrium and activation barriers, which need to be overcome to reach equilibrium, are of the order of magnitude of thermal activation. On the contrary, nanoparticles being around 10 times larger, their dynamics is markedly slower and they easily get trapped in glassy states which do not correspond to a global free en-

ergy minimum. ${ }^{28}$ These out-of-equilibrium configurations can persist indefinitely, compared to experimental time scales because thermal activation energy is not high enough to overcome the activation barriers leading to more stable configurations. Hence, controlling the self-assembly of nanoparticles requires a careful attention to all the steps of the process from the synthesis to the final assembly. Work along these lines is currently in progress in our laboratories.

In conclusion, we have devised a simple way to organize CdSe nanoplatelets into highly anisotropic micronic superparticles through the destabilization of a colloidal solution by addition of an antisolvant. These superparticles are composed of around $10^{6}$ individual nanoparticles and the ordered stacking of the nanoplatelets leads to anisotropic optical properties which could be, in the future, exploited in optoelectronic devices such as LEDs. ${ }^{29}$

\section{Chemicals}

Cadmium acetate dihydrate $\left(\mathrm{Cd}(\mathrm{OAc})_{2} \cdot 2 \mathrm{H}_{2} \mathrm{O}\right)$, technical grade 1-octadecene (ODE), myristic acid sodium salt, oleic acid (OA) and selenium in powder were purchased from Sigma-Aldrich. Ethanol 
and hexane were purchased from SDS Carlo-Erba. The preparation of cadmium myristate was performed as reported elsewhere. ${ }^{30}$

\section{Synthesis and purification of CdSe nanoplatelets}

In a three neck round bottom $25 \mathrm{~mL}$ flask, equipped with a septum, a temperature controller and a condenser, $340 \mathrm{mg}$ of cadmium myristate, $27 \mathrm{mg}$ of selenium powder and $30 \mathrm{~mL}$ of octadecene were degassed under agitation for 30 minutes. Afterwards, the flask was purged with argon and the temperature set-point was set to $240^{\circ} \mathrm{C}$. At $190^{\circ} \mathrm{C}$, the selenium started to dissolve and the solution turned clear and yellow. When the temperature reached $202^{\circ} \mathrm{C}$, which took around 6 min $30 \mathrm{~s}$ with our equipment, the septum was withdrawn and $160 \mathrm{mg}$ of cadmium acetate was swiftly injected in the flask. After the temperature reached $240^{\circ} \mathrm{C}$, the reaction medium was kept under agitation for 8 minutes before $1 \mathrm{~mL}$ of oleic acid was injected. Immediately after this injection, the heating mantle was withdrawn and the flask was cooled with a stream of air. The flask was rinsed with hexane and the crude room temperature product (around $40 \mathrm{~mL}$ ) was divided evenly in two $50 \mathrm{~mL}$ centrifuge tubes. At this point, UV-VIS absorption spectroscopy indicated the presence of nanoplatelets emitting at $462 \mathrm{~nm}$ (3 MLs), nanoplatelets emitting at $513 \mathrm{~nm}$ (4 MLs) and quantum dots. To purify the nanoplatelets, $25 \mathrm{~mL}$ of ethanol was added to each centrifuge tube and centrifugation at $5000 \mathrm{rpm}$ for 10 minutes yielded a yellow pellet and a red supernatant containing only quantum dots. Each pellet was dispersed in $20 \mathrm{~mL}$ of hexane and the solution was centrifuged at $5000 \mathrm{rpm}$ for 10 minutes. The pellet contained only 3 MLs NPs and was discarded while the yellow supernatant contained the 4 MLs NPs. $15 \mathrm{~mL}$ of ethanol was added to both supernatants and centrifugation yielded a yellow pellet, which was dispersed in $20 \mathrm{~mL}$ of hexane. Finally, 15 $\mathrm{mL}$ of ethanol was added to each dispersion and centrifugation at $5000 \mathrm{rpm}$ for 10 minutes results in two yellow pellets, which were both dispersed in a total of $20 \mathrm{~mL}$ of hexane. 


\section{Self-assembly of superparticles}

To obtain the superparticles, $500 \mu \mathrm{L}$ of the nanoplatelet dispersion was first diluted by adding 250 $\mu \mathrm{L}$ of hexane. Aftewards, $250 \mu \mathrm{L}$ of ethanol was added to the previous solution. The quantity of ethanol to be added to the colloidal solution varied from nanoplatelet synthesis batch to batch but the volume fraction in ethanol in the final solution was always comprised between 30 and $50 \%$.

\section{Transmission electron microscopy}

TEM grids were prepared by dropping $10 \mu \mathrm{L}$ of solution on a copper grid covered with a carbon film. Observations were made using a TOPCON electron microscope operated at $100 \mathrm{kV}$.

\section{Small Angle X-ray Scattering}

SAXS experiments were performed at the SWING beamline of the SOLEIL synchrotron (SaintAubin, France). Measurements were carried out using a fixed energy of $12 \mathrm{keV}$ and a sample to detector distance of $1.07 \mathrm{~m}$. The typical accessible range of scattering vector modulus $\mathrm{q}$ was 0.1 to $10 \mathrm{~nm}^{-1}\left(q=\frac{4 \pi}{\lambda} \sin (\theta)\right)$, where $2 \theta$ is the scattering angle and $\lambda=1.033 \AA$ the wavelength). The incident X-ray beam transverse dimensions were approximately $600 \times 200 \mu \mathrm{m}^{2}$ in the horizontal and vertical directions. 2D scattering patterns were recorded on an AVIEX 170170 CCD camera formed by four detectors. The intensity was radially averaged to yield intensity versus q data.

\section{Polarized fluorescence microscopy}

The nanoplatelet supraparticles contained in a thin glass capillary were imaged in epifluorescence through a 60x objective. The fluorescence is separated between the two polarization components which are both imaged on a CCD camera. More details on the optical set-up can be found in ref. ${ }^{31}$ 


\section{Acknowledgements}

B.A. thanks Thomas Pons and Alexandra Fragola for assistance in the polarized fluorescent measurements, Vincent Loriette for a MATLAB code for the treatment of fluorescence images and Mathieu Kociak for assistance during the TEM imaging. We thank the Soleil Synchrotron (SaintAubin, France) for the award of beam-time 20110596 and Florian Meneau for local contact during the SAXS experiments.

\section{Supporting Information}

Supplementary TEM images. This material is available free of charge via the Internet at http://pubs.acs.org.

\section{References}

(1) Nie, Z.; Petukhova, A.; Kumacheva, E. Nature Nanotechnology 2010, 5, 15-25.

(2) Glotzer, S. C.; Solomon, M. J. Nature Materials 2007, 6, 557-562.

(3) Gupta, S.; Zhang, Q.; Emrick, T.; Russell, T. P. Nano Lett. 2006, 6, 2066-2069.

(4) Baranov, D.; Fiore, A.; van Huis, M.; Giannini, C.; Falqui, A.; Lafont, U.; Zandbergen, H.; Zanella, M.; Cingolani, R.; Manna, L. Nano Lett. 2010, 10, 743-749.

(5) Talapin, D. V.; Shevchenko, E. V.; Murray, C. B.; Kornowski, A.; Forster, S.; Weller, H. J. Am. Chem. Soc. 2004, 126, 12984-12988.

(6) Baker, J. L.; Widmer-Cooper, A.; Toney, M. F.; Geissler, P. L.; Alivisatos, A. P. Nano Lett. 2010, 10, 195-201.

(7) Miszta, K.; de Graaf, J.; Bertoni, G.; Dorfs, D.; Brescia, R.; Marras, S.; Ceseracciu, L.; Cingolani, R.; van Roij, R.; Dijkstra, M.; Manna, L. Nature Materials 2011, 10, 872-876.

(8) Wang, T.; Zhuang, J.; Lynch, J.; Chen, O.; Wang, Z.; Wang, X.; LaMontagne, D.; Wu, H.; Wang, Z.; Cao, Y. C. Science 2012, 338, 358-363. 
(9) Wang, T.; LaMontagne, D.; Lynch, J.; Zhuang, J.; Cao, Y. C. Chem. Soc. Rev. 2013, 42, 2804-2823.

(10) Ithurria, S.; Dubertret, B. J. Am. Chem. Soc. 2008, 130, 16504-16505.

(11) Ithurria, S.; Bousquet, G.; Dubertret, B. J. Am. Chem. Soc. 2011, 133, 3070-3077.

(12) Ithurria, S.; Tessier, M. D.; Mahler, B.; Lobo, R. P. S. M.; Dubertret, B.; Efros, A. L. Nature Materials 2011, 10, 1-6.

(13) Mahler, B.; Nadal, B.; Bouet, C.; Patriarche, G.; Dubertret, B. J. Am. Chem. Soc. 2012, 134, $18591-18598$.

(14) Jones, M. R.; Macfarlane, R. J.; Prigodich, A. E.; Patel, P. C.; Mirkin, C. A. J. Am. Chem. Soc. 2011, 133, 18865-18869.

(15) Glotzer, S. C. Nature 2012, 481, 450-452.

(16) Paik, T.; Ko, D.-K.; Gordon, T. R.; Doan-Nguyen, V.; Murray, C. B. ACS Nano 2011, 5, 8322-8330.

(17) Ye, X.; Chen, J.; Engel, M.; Millan, J. A.; Li, W.; Qi, L.; Xing, G.; Collins, J. E.; Kagan, C. R.; Li, J.; Glotzer, S. C.; Murray, C. B. Nature Chemistry 2013, 1-8.

(18) Saunders, A. E.; Ghezelbash, A.; Smilgies, D.-M.; Sigman, M. B.; Korgel, B. A. Nano Lett. 2006, 6, 2959-2963.

(19) Du, X. S.; Mo, M.; Zheng, R.; Lim, S. H.; Meng, Y. Cryst. Growth Des. 2008, 8, 2032-2035.

(20) Tessier, M. D.; Biadala, L.; Bouet, C.; Ithurria, S.; Abecassis, B.; Dubertret, B. ACS Nano 2013, 7, 3332-3340.

(21) Hu, J.; Li, L.-S.; Yang, W.; Manna, L.; Wang, L.-W.; Alivisatos, A. P. Science 2001, 292, 2060-2063. 
(22) Hassinen, A.; Moreels, I.; De Nolf, K.; Smet, P. F.; Martins, J. C.; Hens, Z. J. Am. Chem. Soc. 2012, 134, 20705-20712.

(23) Godfrey Alig, A. R.; Akbulut, M.; Golan, Y.; Israelachvili, J. Adv. Funct. Mater. 2006, 16, $2127-2134$.

(24) Min, Y.; Akbulut, M.; Prud'homme, R. K.; Golan, Y.; Israelachvili, J. J. Phys. Chem. B 2008, $112,14395-14401$.

(25) Zang, L.; Che, Y.; Moore, J. S. Acc. Chem. Res. 2008, 41, 1596-1608.

(26) Che, Y.; Datar, A.; Balakrishnan, K.; Zang, L. J. Am. Chem. Soc. 2007, 129, 7234-7235.

(27) Datar, A.; Balakrishnan, K.; Yang, X.; Zuo, X.; Huang, J.; Oitker, R.; Yen, M.; Zhao, J.; Tiede, D. M.; Zang, L. J. Phys. Chem. B 2006, 110, 12327-12332.

(28) Min, Y.; Akbulut, M.; Kristiansen, K.; Golan, Y.; Israelachvili, J. Nature Materials 2008, 7, $527-538$.

(29) Chen, Z.; Nadal, B.; Mahler, B.; Aubin, H.; Dubertret, B. Adv. Funct. Mater., in press.

(30) Yang, Y. A.; Wu, H.; Williams, K. R.; Cao, Y. C. Angew. Chem. 2005, 117, 6870-6873.

(31) Cassette, E.; Mahler, B.; Guigner, J.-M.; Patriarche, G.; Dubertret, B.; Pons, T. ACS Nano 2012, 6, 6741-6750. 


\section{For TOC only}

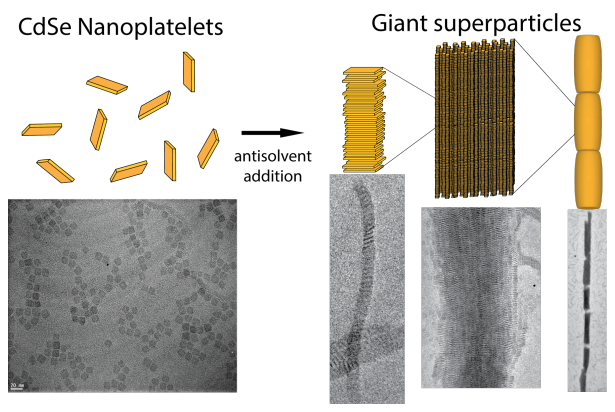

